\title{
Effects of generalization of fear on incubation in a response suppression paradigm
}

\author{
LAWRENCE G. KORANDA and P. E. FREEDMAN \\ University of Illinois at Chicago Circle, Chicago, Ilinois 60680
}

\begin{abstract}
Seventy-two rats were run in a suppression paradigm in one of 12 combinations of delay after fear conditioning ( 0 vs. $23^{1 / 2}$ h), mode of fear conditioning (forward, backward, and CS-only control), and similarity between fear conditioning and testing chambers (same vs. different). The forward fear conditioning groups showed incubation (greater suppression after the delay) under both same and different conditions. This finding does not support a stimulus generalization interpretation of the incubation effect.
\end{abstract}

The term "incubation" of fear refers to the "growth of fear over a time interval which follows some aversive stimulation .... and the time interval is free of further exposure to the aversive stimuli" (McAllister \& McAllister, 1967, p. 180). The literature contains two studies which used "incubation" to account for increasing suppression of a positively reinforced response by a CS previously paired with shock as a direct function of increasing time between exposure to shock and testing for fear conditioned to the CS.

McMicheal (1966) investigated the amount of fear elicited by a tone CS that had been paired with footshock. Fear conditioning took place in a shuttlebox, while suppression was measured in an operant box. The effect of postconditioning delay was significant, with suppression of barpressing in the presence of the CS increasing from .03 to $6 \mathrm{~h}$, then leveling off at 24, 48, and $504 \mathrm{~h}$. The results were interpreted as demonstrating an increase in fear as a function of time between fear conditioning and testing. The increase was attributed to the "incubation" of fear.

Tarpy (1966) replicated the avoidance conditioning portion of the McMicheal study. Fear conditioning occurred in a tilt cage and involved avoidance training with a buzzer CS and footshock UCS. Subjects were tested in an operant box. The results showed significantly greater suppression of responding at 4,24 , and $168 \mathrm{~h}$ than at 0 or $1 \mathrm{~h}$ in the first $10 \mathrm{~min}$ of CS testing. No significant differences in suppression were found during the second $10 \mathrm{~min}$ of testing. The interpretation was that incubation of fear had been responsible for the observed increases in response suppression. It should be noted here that, in both of the above studies, the environment for fear conditioning was different from the environment for testing.

McAllister and McAllister (1967) have objected to the "incubation" interpretation on the basis that another equally plausible explanation of the results could be made. Their alternative explanation is based on generalization. According to this view, the fear conditioned to the stimuli present during fear conditioning (the discrete
CS and apparatus stimuli) generalizes to the stimuli present in the test situation. The degree of generalization determines the amount of fear elicited by the testing stimuli. A high degree of generalization would result in greater fear in testing than lower degrees. In any case, the amount of fear elicited by the generalized stimuli would presumably not exceed that elicited by the original fear conditioning situation. Two variables, stimulus similarity between fear conditioning and testing situations and the length of postconditioning delay, determine the degree of generalization. If fear conditioning and testing occur in dissimilar situations, there would be a low degree of stimulus generalization (generalization decrement) at short postconditioning delay intervals. With longer postconditioning delays, there is an increase in the degree of generalization. The testing stimuli would elicit more fear at long delays than at shorter delays. If fear conditioning and testing occur in the same or highly similar situations, the degree of generalization is already at a high level. Hence, the fear elicited in testing is at a maximal level and cannot be increased further. Increased postconditioning delay does not increase the degree of generalization in this instance.

Support for this hypothesis comes from research utilizing hurdle jumping escape (Desiderato, Butler, \& Meyer, 1966; McAllister \& McAllister, 1962a, 1963, 1965) and straight alley running speed (Saltz \& Asdourian, 1963). Increase in performance as a function of increases in the length of postconditioning delay were found only when the conditioning and testing situations were dissimilar. When the situations were the same or highly similar, subjects showed the same level of fear regardless of the length of postconditioning delay.

As was previously mentioned, the generalization hypothesis states that the relevant stimuli involved are the discrete CS and apparatus cues. McAllister and McAllister (1962b) performed two experiments to determine the amount of fear conditioned to each of these components separately. Following forward or backward CS fear conditioning in a neutral box, they tested hurdle jump escape with or without a CS present, or with or 
without extinction exposure in the hurdle jump apparatus. Escape was quickest in the CS forward condition, where both CS and generalized apparatus cues were utilized. However, in groups where the CS was omitted in testing, escape was learned, indicating fear generalized to apparatus cues. Finally, when subjects were confined in the hurdle jump startbox, allowing extinction of generalized fear, hurdle jumping escape was not acquired.

McAllister and McAllister (1967) have suggested that the generalization hypothesis can account for the response suppression effects found in the McMicheal (1966) and Tarpy (1966) studies. As was noted, in both studies fear conditioning and testing had occurred in different pieces of apparatus. It is the purpose of the present study to test the applicability of the generalization hypothesis to the response suppression paradigm, by varying both delay and apparatus cues.

\section{METHOD}

\section{Subjects}

The subjects were 72 Charles River male albino rats, 100-120 days old, housed in individual home cages. They were kept on a $231 / 2-h$ food deprivation schedule, with free access to water throughout the experiment.

\section{Apparatus}

Two major types of apparatus were used. The first was two commercial operant boxes, $30 \times 21 \times 34 \mathrm{~cm}$, with Plexiglas side walls and tops, and metal back walls. On the metal front wall in each box was a $2.5 \times 1.3 \mathrm{~cm}$ metal bar located $2.5 \mathrm{~cm}$ from the floor and $2.0 \mathrm{~cm}$ from the right side wall. A metal foodcup was also on the front wall $5.0 \mathrm{~cm}$ left of the bar and $1.3 \mathrm{~cm}$ from the floor. The foodcup received the reinforcement, a single $45-\mathrm{mg}$ Noyes food pellet, delivered by a pellet feeder. The floors consisted of $.5-\mathrm{cm}$-diam stainless steel rods spaced $1.3 \mathrm{~cm}$ apart and wired to deliver shock. The box-feeder combinations were each enclosed in a sound-deadened chamber equipped with light, ventilator fan, and speaker which delivered the tone CS $(1,175 \mathrm{~Hz}$ of moderate intensity).

The second type of apparatus was the right-hand compartment $(30 \times 20 \times 27 \mathrm{~cm})$ of a bidirectional shuttlebox. The front was Plexiglas, the top and back walls were wood, and the side walls were metal. The floor, .4-cm-diam stainless steel rods spaced $1.3 \mathrm{~cm}$ apart, was wired to deliver shock. The tone CS was presented through a speaker mounted in the top of the shuttlebox compartment. The entire apparatus was enclosed in a sound-deadened chamber with light and ventilator fan.

Additional equipment consisted of two BRS Model SGS 001 shock generator scramblers set to deliver a .1-W UCS (a level used in our laboratory for high-intensity shock). Other automated equipment controlled reinforcement schedules, CS and UCS presentation, and data recording.

\section{Procedure}

All subjects were maintained on a $231 / 2$-h food deprivation schedule, and were shaped to barpress for food on a VI .5-min schedule. This training occurred in the operant boxes in daily $1 / 2$-h sessions with both CS and UCS absent. For each subject, the number of barpresses in $301-$ min intervals was recorded and daily totals were computed. Daily training sessions continued until subjects achieved stable performance over 3 consecutive days of barpressing. The daily totals of the last 2 days in the 3-day series were compared to the first daily total. If the last 2 days were within $20 \%$ of the first day, then performance was considered stable.

After subjects had achieved stability, they were randomly assigned to the 12 cells of the experimental design, resulting in six subjects per cell. Each cell designated subjects' treatment with respect to place of fear conditioning, SAME or DIFF (operant box or shuttlebox); delay between fear conditioning and testing, 0 or $231 / 2 \mathrm{~h}$; and type of fear conditioning, forward conditioning (F), backward conditioning (B), or nonshock control (C). Fear conditioning occurred at the time normally scheduled for a VI barpress session. No bar was present in the operant boxes during fear conditioning. Subjects in the F condition had 15 CS-UCS pairings, with the 30 -sec CS preceding the 1-sec UCS coterminating. The mean ITI was 90 sec. The subjects in the B condition received 15 CS-UCS pairings in which the 1 -sec UCS preceded the 30 -sec CS by an average of $15 \mathrm{sec}$, with a mean ITI of $74 \mathrm{sec}$. The C condition consisted of 15 presentations of the 30 -sec CS only, with a mean ITI of 90 sec. Subjects were then removed from the fear conditioning apparatus and placed in their home cages for the appropriate postconditioning delay interval.

After the appropriate delay had elapsed, subjects were returned to the operant boxes for a $30-\mathrm{min}$ testing session. For all subjects during testing, the bar was restored and the VI .5-min schedule was in effect, with food reinforcement available. No UCS was presented during testing. Testing consisted of three consecutive 10-min periods, labeled Test Periods I, II, and III. In the first $10 \mathrm{~min}$ of testing (I), no CS was presented. Number of responses was recorded in 30 -sec intervals to provide a measure of postshock influence of fear conditioned to apparatus cues on barpressing. In the remaining $20 \mathrm{~min}$ (II and III), the CS was randomly presented 10 times, each presentation of $30-\mathrm{sec}$ duration, with an average of $90 \mathrm{sec}$ between presentations. Number of responses in the 30 preceding the CS, the $30 \mathrm{sec}$ during the CS, and during the average 30 -sec ITI were recorded. In addition, visual observations were made of subjects' behavior in testing.

Subjects were run in squads of 12 animais each. One squad was trained, fear conditioned, and tested before another squad of subjects was run.

\section{RESULTS}

Suppression ratios were computed separately for each subject from the data in Test Periods II and III, during which the CS used in fear conditioning was randomly presented. The ratios were calculated by the formula (Kamin, 1963) A/B + A, with A being the number of responses during the $30 \mathrm{sec}$ when the CS was on and $B$ being the number of responses in the 30-sec interval immediately preceding the onset of the CS. As an index of conditioned fear, a smaller value of the suppression ratio (greater suppression) indicates a higher level of conditioned fear than larger values (less suppression).

\section{Suppression Ratios}

Figures 1 and 2 show the suppression ratios for the 12 groups in the experiment during Test Periods II and III, the CS "on" test periods. Figure 1 is for the six groups, two conditions of Delay ( 0 and $23 \frac{1}{2} \mathrm{~h}$ ) by three fear conditioning treatments (F-Forward conditioning, B-Backward conditioning, and C-CS only Controls), which were fear conditioned and tested in the same place, the operant box. Figure 2 is for the six groups in the Different place treatment.

The 12 independent groups permitted an analysis of variance using 11 single degree-of-freedom planned comparisons to test for between-group differences. The data for Test Periods II and III were analyzed separately. 


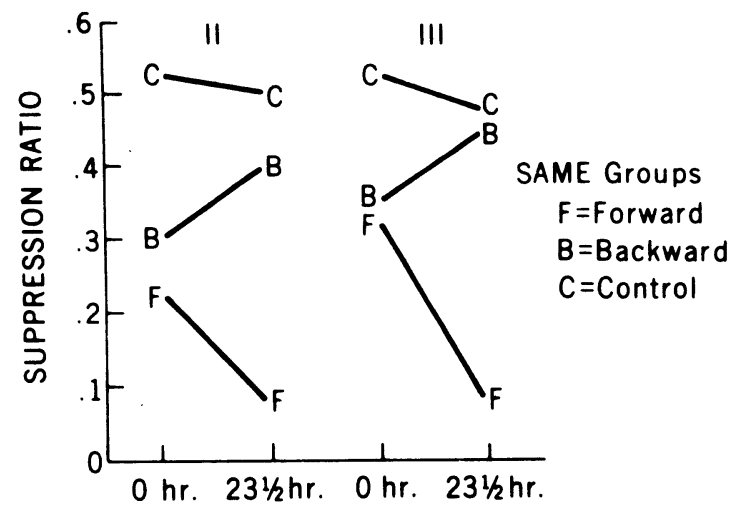

Figure 1. Suppression ratios in Test Period II and III for six groups (0- and 231/2-h Delay by Forward, Backward, and Control Type of fear conditioning) fear conditioned and tested in the SAME place. $Y$ axis is response suppression ratio. $X$ axis is delay ( 0 and $23 \frac{1}{2} \mathrm{~h}$ ).

From Figures 1 and 2, it appears that Forward conditioned groups showed smaller suppression ratios than Backward conditioned groups. The analysis of variance between the overall means of Forward groups in Test Periods II and III, .136 and .207, and Backward groups in these two test periods, .373 and .449 , confirmed this observation with $\mathrm{F}(1,60)=25.79(\mathrm{p}<.01)$ in Period II and $F(1,60)=39.40(p<.01)$ in Period III. Backward conditioned subjects differed significantly from nonshocked Control groups (mean of .515) only in Test Period II, $F(1,60)=5.98(p<.02)$. In Test Period III, Backward groups did not differ from Controls, means of .449 vs. $.512, F(1,60)=1.03$. These results were taken as indicating a significant main effect of Type of fear conditioning.

The analysis of variance revealed an overall significant effect of the Delay treatment imposed between fear conditioning and testing. In Test Period II, the mean of the 0-h Delay groups (.288) was significantly larger than the mean of the $231 / 2-\mathrm{h}$ Delay groups (.221), with an $\mathrm{F}(1,60)=12.51(\mathrm{p}<.01)$. In Test Period III the planned comparison between the $0-\mathrm{h}$ and $23 \frac{1}{2}-\mathrm{h}$ Delay mean, .353 vs. .303 , produced a significant $F(1,60)$ of 6.92 $(\mathrm{p}<.02)$.

Although the significant main effect of Delay supports the conclusion that, overall, subjects showed smaller suppression ratios at $231 / 2 \mathrm{~h}$ than at $0 \mathrm{~h}$, Figures 1 and 2 suggest that only Forward conditioned groups showed a decline in barpressing as a direct function of Delay. Backward conditioned groups apparently increased barpressing from $0-h$ to $23 \frac{1}{2}-\mathrm{h}$ Delay. The significant interaction of Delay by Type of fear conditioning in Test Period II $[\mathrm{F}(1,60)=4.75, \mathrm{p}<.05]$ and III $[F(1,60)=11.07, p<.01]$ led to post hoc examination of the data to determine the source of these interactions.

The four groups involved in a Scheffe post hoc comparison were the Forward-0 h, Forward $-23 \frac{1}{2} \mathrm{~h}$, Backward $-0 \mathrm{~h}$, and Backward-231/2 h. The Scheffe test established that Test Period II means must differ by at least
.1779 to be significant at the .05 level. Only the mean of the Forward-231/2 $\mathrm{h}$ group (.045) differed significantly from the means of the Forward-0 $\mathrm{h}(.2276)$ by .1827 , Backward-0 h (.3472) by .3023 , and Backward-231/2 h (.3981) by .3532 . These latter three groups had a maximum mean difference of only .1705 and were not statistically different from each other. In Test Period III similar results were obtained. In this test period the range for a .05 significance level was .1723 . Since the Backward groups previously had been found not to differ from Controls, the comparison was between the means of the Forward-0 h (.294) and Forward-231/2h (.120). The difference between these means was .1740, a significant difference. Therefore, the major factor contributing to the significant main effect of Delay and the Delay by Type interaction was the decline in barpressing of Forward groups tested at $231 / 2 \mathrm{~h}$ as compared to Forward groups tested at $0 \mathrm{~h}$. Backward groups either showed no difference between 0 and $23 \frac{1}{2} \mathrm{~h}$ or were not different from Control groups.

A comparison between the results presented in Figure 1 and Figure 2, Same Place vs. Diff Place of fear conditioning and testing, showed no significant main effect of this treatment. Other two-way and three-way interactions were not significant.

The nonshocked control groups were not all equal. The control group which received CS-only presentations in the operant box and a Delay treatment of $231 / 2 \mathrm{~h}$ (Same-231/2-Control) had Period II and III ratios of .505 and .474 . These ratios were different from the Same-0Control means of .533 and $.522[\mathrm{Fs}(1,60)=12.57$, $\mathrm{p}<.01$ and $4.52, \mathrm{p}<.05$ ] . These ratios for the Same-0Control were different than the Diff-0-Control means in Test Periods II and III $(.517$ and .523$), F(1,60)=48.49$ $(\mathrm{p}<.01)$ and $\mathrm{F}(1,60)=33.44(\mathrm{p}<.01)$. The Diff-0Control group was not different than the Diff- $23 \frac{1}{2}$ Control group.

Since no CS was presented during the first 10 min of testing (Test Period I), it was not possible to compute a suppression ratio for this period. The measure for each

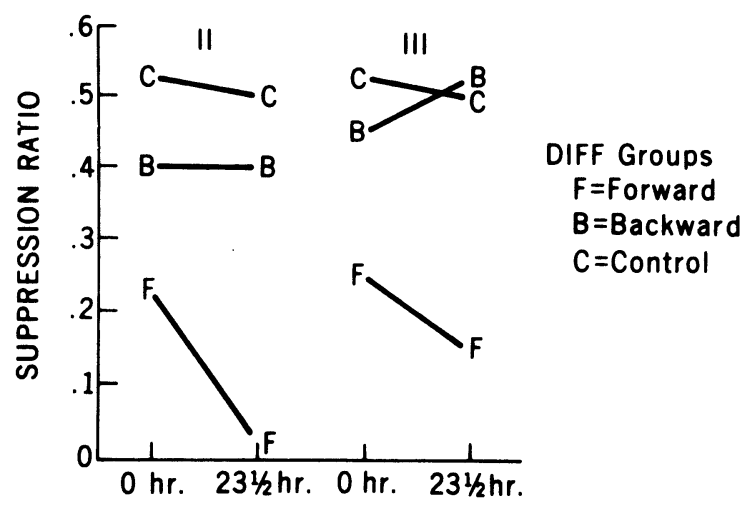

Figure 2. Suppression ratios in Test Periods II and III for six groups (0- and 231/2-h Delay by Forward, Backward, and Control Type of fear conditioning) fear conditioned and tested in the DIFF place. $Y$ axis is response suppression ratio. $X$ axis is delay $(0$ and $231 / 2 h)$. 
subject was the percentage of a corresponding baseline period prior to fear conditioning. The formula was: Percentage $=$ Test $I /$ Training $I$, where Test $I$ was the number of barpresses in the first $10 \mathrm{~min}$ of testing. Training I was the mean number of barpresses during the first $10 \mathrm{~min}$ of the 3 days immediately preceding fear conditioning. No significant differences were found between groups during the first $10 \mathrm{~min}$ of testing.

\section{DISCUSSION}

The results of the present study supported the prediction that subjects shocked and tested in a different apparatus would show a significant positive effect of variations in postconditioning delay. The findings for subjects in the DIFF-F treatment agree with this prediction. The groups which received forward fear conditioning in the shuttlebox and were tested in the Skinner box showed significantly greater fear (fewer responses) at $231 / 2 \mathrm{~h}$ than at $0 \mathrm{~h}$. Similar results have been obtained by other studies using response suppression and the escape-from-fear paradigm (McMicheal, 1966; Tarpy, 1966). Tarpy (1966) also reported that there were no significant differences between groups after the initial CS exposure period. The present study found differences in both Test Periods II and III. The studies using the escape-from-fear paradigm, McAllister and McAllister (1962a, 1963, 1965) and Desiderato, Butler, and Meyer (1966), all had some conditions in which subjects were given forward fear conditioning trials in an apparatus different than that used in testing. Each of these studies reported increased performance as a function of postconditioning delay.

The results of the present study failed to support the prediction that, if fear conditioning and testing occurred in the same apparatus, then no effect of postconditioning delay would be seen. The present study found that subjects in SAME- $t$ treatment did show increased suppression at the longer delay of $231 / 2 \mathrm{~h}$ compared to the 0 - $\mathrm{h}$ delay. The McAllister and McAllister $(1962 a, 1963,1965)$ and Disiderato, Butler, and Meyer (1966) studies also contained conditions in which subjects were fear conditioned and tested in the same apparatus. These studies reported no changes in performance across intervals of postconditioning delay.

The results of this experiment found no significant differences between SAME and DIFF treatments as a function of delay. A possible explanation for this finding may be that, with respect to several important stimuli, fear conditioning and testing situations were not identical. One important stimulus was the bar in the Skinner box. The importance of this cue can be inferred from the visual observations of subjects' behavior in training, fear conditioning, and testing. Upon placement in the Skinner box, subjects would orient to the bar area prior to bar extension. Immediately after bar extension, subjects would begin pressing. During fear conditioning in the Skinner box, the bar was not extended. Subjects would initially orient to the bar area and remain in that position until the CS and UCS onset. In testing, subjects would again face the bar area and begin pressing after bar extension. Therefore, the test situation had a distinctly different stimulus component (the bar) than the fear conditioning situation (no bar). Other stimuli which were absent during fear conditioning and present during testing were the pellet feeder clicks and the sound of pellet delivery. Hence, it is possible that, although fear conditioning and testing occurred in the same apparatus, the stimuli present on each occasion were different.

Another discrepant result concerns the influence of apparatus stimuli in testing. In the first $10 \mathrm{~min}$ of testing (I), the only stimuli present were the apparatus stimuli. During this period, no differences between groups were found. McAllister and McAllister (1962b) had demonstrated that the apparatus stimuli present during UCS onset could elicit fear in testing. The failure of the present study to replicate this finding may be due to one or both of the following factors. First, fear conditioned to the apparatus stimuli could have extinguished during the intertrial interval in fear conditioning. A second possible factor is that during barpress training the apparatus stimuli present in the Skinner box were paired with an appetitive stimulus (food). Thus, these stimuli would have positive as well as aversive qualities in testing. It may have been the case that the appetitive aspects of the apparatus stimuli offset their aversive effects.

McAllister and McAllister (1962b) used a backward fear conditioning procedure with a hurdle jumping test. Kamin (1963) also used backward fear conditioning with a response suppression paradigm as a test for conditioned fear. The results of both studies demonstrated that the discrete CS had remained neutral. The findings of the present study revealed a significant decrease in responding during the CS in Test Period II for the B groups, in comparison to nonshocked controls. The magnitude of this decrease was less than that for forward conditioned subjects. Furthermore, backward conditioned groups showed no difference from controls in Period III and no further response decrements with increased postconditioning delay. The initial suppression of responding in the presence of the tone (CS) may have resulted from pseudoconditioning. Or the CS might have been a cue for the B subjects which indicated a return to a situation (fear conditioning) in which shock occurred.

These findings of the present study do not support a generalization of fear interpretation. Most notably, subjects showed increased suppression of responding, with corresponding increases in postconditioning delay independent of the apparatus that had been used for fear conditioning. The hypothesis which predicts such increases is the "incubation" of fear. Yet, the possibility of stimulus differences between fear conditioning and testing in the same situation must be investigated before "incubation" can be firmly established as a primary explanation of observed performance decreases as a direct function of postconditioning delay.

\section{REFERENCES}

Desiderato, O., Butler, B., \& Meyer, C. Changes in fear generalization gradients as a function of delayed testing. Journal of Experimental Psychology, 1966, 72, 678-682.

KAMIN, L. J. Backward conditioning of the conditioned emotional response. Journal of Comparative and Physiological Psychology, 1963, 56, 517-519.

McAllister, D. E., \& MCAllister, W. R. Incubation of fear: An examination of the concept. Journal of Experimental Research in Personality, 1967, 2, 191-199.

McAllister, W. R., \& McAllister, D. E. Increase over time of the stimulus generalization of fear. Journal of Experimental Psychology, 1963, 65, 576-582. (a)

McAllister, W. R., \& McAllister, D. E. Role of the CS and apparatus cues in the measurement of acquired fear. Psychological Reports, 1962, 11, 749-756. (b)

MCAllister, W. R., \& McAllister, D. E. Variables influencing the conditioning and measurement of acquired fear. In W. F. Prokasy (Ed.), Classical conditioning: A symposium. New York: Appleton-Century-Crofts, 1965. Pp. 172-191.

McMicheal, J. S. Incubation of anxiety and instrumental behavior. Journal of Comparative and Physiological Psycho$\log y, 1966,61,208-211$.

SAltz, E., \& Asdourian, D. Incubation of anxiety as a function of cognitive differentiation. Journal of Experimental Psychology, 196j, 66, 17-22.

TARPY, R. M. Incubation of anxiety as measured by response suppression. Psychonomic Science, 1966, 4, 189-190. 